\title{
COVID-19 Vaccination Considerations in Pregnant Women
}

\author{
Masoud Mardani (iD ${ }^{1}$ and Bita Pourkaveh (iD ${ }^{2,{ }^{*}}$ \\ ${ }^{1}$ Infectious Diseases and Tropical Medicine Research Center, Shahid Beheshti University of Medical Sciences, Tehran, Iran \\ ${ }^{2}$ Anatomy and Biology Department, Shahid Beheshti University of Medical Sciences, Tehran, Iran \\ Corresponding author: Biology and Anatomy Department, Faculty of Medicine, Shahid Beheshti University of Medical Sciences, Tehran, Iran. Email: \\ bitapourkaveh@yahoo.com \\ Received 2021 April 10; Accepted 2021 April 10.
}

Keywords: Pregnancy, Vaccination, COVID-19

Pregnant women with severe coronavirus disease 2019 (COVID-19) infection are at an increased risk for severe illness, preterm delivery, and pregnancy loss. In a cohort study, 252 SARS-CoV-2-positive and 3122 healthy pregnant women were studied, which showed that infected pregnant women are at risk for iatrogenic and spontaneous preterm delivery, the incidence of which ranged from $10 \%$ to $25 \%$, and the rate of severe disease in SARS-CoV-2-positive pregnant women was as high as $60 \%$ (1).

Although data regarding pregnancy is still emerging, but it seems that pregnant women in comparison with non-pregnant women are at a greater risk for severe illness and mortality caused by COVID-19. This primary risk may be related to the immunological changes during pregnancy. In an analysis of 409,462 pregnant women with symptomatic COVID-19, the adjusted risk ratio in pregnant women versus non-pregnant was 3.0 for intensive care unit admission, 2.9 for mechanical ventilation, and 1.7 for death $(2,3)$. Thus, both the mother and the fetus should be kept away from serious complications of the coronavirus.

Although it is unknown whether the mother's immune response protects the fetus against the infection or not, it has been found that neonatal infections are very rare. Considering that COVID-19 IgG has been observed in neonates with negative IgM and negative polymerase chain reaction (PCR), it can be suggested SARS-CoV-2-specific antibodies are effectively transferred across the placenta following third-trimester maternal infection, similar to what happens in influenza and pertussis. One of the effective ways to prevent maternal infections and infant morbidity during pregnancy is vaccination, similar to influenza and pertussis vaccination, which is highly recommended, and there is abundant, supportive clinical data on its safety and efficacy (3).

mRNA platforms that are being utilized for the devel- opment of COVID-19 vaccines are completely different from influenza and Tdap vaccines used during pregnancy. Also, mRNA vaccines have been used in Zika vaccine and vaccines for cancers such as breast cancer and melanoma.

It is clear that mRNA platforms are immunogenic and noninfectious and enjoy potential benefits over live attenuated, inactivated, subunit, and DNA-based vaccines. Also, there is no evidence that nanoparticle vaccines can reach the fetus following vaccination, but it seems that immune response stimulation is the result of taking up the lipid nanoparticles by local muscle cells and subsequent transcription initiation (3).

The Center for Disease Control and the independent Advisory Committee on Immunization Practices (ACIP) have provided information to help pregnant women with their decision to receive the COVID-19 vaccine. Meanwhile, Participation of pregnant women in the first month of the COVID-19 vaccination program, similar to other specific groups such as health care personnel, is currently recommended by ACIP. However, the information regarding COVID-19 vaccines (mRNA vaccines included) administered during pregnancy is limited. Even limited data is currently at hand from animal developmental and reproductive toxicity studies. Besides, Moderna COVID-19 vaccine has been studied in rats, and no safety concerns have been observed before or during pregnancy; studies of the Pfizer-BioNTech vaccine are ongoing (4).

Safety monitoring programs are in progress by the CDC and the Food and Drug Administration (FDA) to collect data regarding vaccination during pregnancy (4). Moreover, there is a global study of 400024 to 34-week pregnant women to survey the safety, tolerability, and immunogenicity of the Pfizer/BionTech vaccine during pregnancy, which will be followed by a six-months study of the safety and passage of protective antibodies to the infant. 
Given that mRNA vaccines do not contain the live virus that causes COVID-19, it cannot give one COVID-19. Also, mRNA does not enter the nucleus of the cell, so it does not interact with a person's DNA and will be quickly broken down by cells. Based on these facts, experts are of the opinion that mRNA vaccines do not pose a specific risk for pregnant women. On the other hand, there are not any comprehensive studies investigating the actual risks of mRNA vaccines to pregnant women and their fetuses; thus, their effects and possible side effects remain unknown (4).

Owing to the fact that the guidance from professional societies and agencies are limited, an explicit recommendation for COVID-19 vaccination in pregnancy is not available. Although the WHO, on January 26, 2021, recommended against vaccination of pregnant women using the Moderna vaccine, except in select circumstances, the American College of Obstetricians and Gynecologists and the Society for Maternal-Fetal Medicine advocate making COVID-19 vaccines available to pregnant and lactating women. Then, the WHO statement 8 was revised on January 29, 2021, that gives permission to high-risk pregnant women (health care workers) or those with comorbidities that add to their risk of severe disease to be vaccinated in consultation with their health care provider (3).

Data on the safety of COVID-19 vaccines in lactating women and the effects of mRNA vaccines on breastfed infants or on milk production/excretion is limited. It seems that mRNA vaccines are not thought to be a risk to breastfeeding infants; therefore, it is recommended that breastfeeding women who are among the groups recommended receiving a COVID-19 vaccine, such as healthcare personnel, may choose to be vaccinated $(4,5)$.

\section{Footnotes}

Authors' Contribution: Not declared by the authors. Conflict of Interests: None declared.

\section{References}

1. Adhikari EH, Moreno W, Zofkie AC, MacDonald L, McIntire DD, Collins RRJ, et al. Pregnancy outcomes among women with and without severe acute respiratory syndrome coronavirus 2 infection. JAMA Netw Open. 2020;3(11). e2029256. doi: 10.1001/jamanetworkopen.2020.29256. [PubMed: 33211113]. [PubMed Central: PMC7677755].

2. Zambrano LD, Ellington S, Strid P, Galang RR, Oduyebo T, Tong VT, et al. Update: Characteristics of symptomatic women of reproductive age with laboratory-confirmed SARS-CoV-2 infection by pregnancy status - United States, January 22-October 3, 2020. MMWR Morb Mortal Wkly Rep. 2020;69(44):1641-7. doi: 10.15585/mmwr.mm6944e3. [PubMed: 33151921]. [PubMed Central: PMC7643892].

3. Adhikari EH, Spong CY. COVID-19 vaccination in pregnant and lactating women. JAMA. 2021;325(11):1039-40. doi: 10.1001/jama.2021.1658. [PubMed: 33555297].

4. CDC. Information about COVID-19 vaccines for people who are pregnant or breastfeeding. 2021, [updated May 14, 2021]. Available from: https://www.cdc.gov/coronavirus/2019-ncov/vaccines/ recommendations/pregnancy.html.

5. Pardi N, Hogan MJ, Porter FW, Weissman D. mRNA vaccines a new era in vaccinology. Nat Rev Drug Discov. 2018;17(4):261-79. doi: 10.1038/nrd.2017.243. [PubMed: 29326426]. [PubMed Central: PMC5906799]. 